\title{
Designing Crystallization to Tune the Performance of Phase-Change Memory: Rules of Hierarchical Melt and Coordinate Bond
}

\section{Jin Zhao}

Shanghai Institute of Microsystem and Information Technology

\section{Wenxiong Song}

Shanghai Institute of Microsystem and Information Technology https://orcid.org/0000-0003-07545786

\section{Tianjiao Xin}

Shanghai Institute of Microsystem and Information Technology

\section{Zhitang Song ( $\nabla$ ztsong@mail.sim.ac.cn )}

Shanghai Institute of Microsystem and Information Technology

\section{Article}

Keywords: Alloy Design, Alloy-tuned Crystallization, Direct Atomic-level Evidence, Storage-class Memory

Posted Date: December 14th, 2020

DOl: https://doi.org/10.21203/rs.3.rs-118565/v1

License: (c) (i) This work is licensed under a Creative Commons Attribution 4.0 International License. Read Full License

Version of Record: A version of this preprint was published at Nature Communications on November 9th, 2021. See the published version at https://doi.org/10.1038/s41467-021-26696-9. 


\title{
Designing Crystallization to Tune the Performance of Phase-Change Memory: Rules of Hierarchical Melt and Coordinate Bond
}

\author{
Jin Zhao ${ }^{1,2,3}$, Wen-Xiong Song ${ }^{1^{*}}$, Tianjiao Xin ${ }^{1}$, Zhitang Song ${ }^{1 *}$
}

\begin{abstract}
${ }^{1}$ State Key Laboratory of Functional Materials for Informatics, Shanghai Institute of Microsystem and Information, Chinese Academy of Sciences, Shanghai 200050, China; ${ }^{2}$ School of Physical Science and Technology, Shanghai Tech University, Shanghai 201210, China; ${ }^{3}$ University of Chinese Academy of Sciences, Beijing, 100049, China.
\end{abstract}

While alloy design has practically shown an efficient strategy to mediate two seemingly conflicted performances of writing speed and data retention in phase-change memory, the detailed kinetic pathway of alloy-tuned crystallization is still unclear. Here, we propose hierarchical melt and coordinate bond strategies to solve them, where the former stabilizes a medium-range crystal-like region and the latter provides a rule to stabilize amorphous. $\mathrm{The}^{\mathrm{E}} \mathrm{r}_{0.52} \mathrm{Sb}_{2} \mathrm{Te}_{3}$ compound we designed achieves writing speed of $3.2 \mathrm{~ns}$ and ten-year data retention of $161^{\circ} \mathrm{C}$. We provide a direct atomic-level evidence that two neighbor Er atoms stabilize a medium-range crystal-like region, acting as a precursor to accelerate crystallization; meanwhile, the essential reason of stabilization originates from the formation of coordinate bonds by sharing lone-pair electrons of chalcogenide atoms with the empty $d$ orbitals of Er atoms. The two rules pave the way for the development of storage-class memory with excellent comprehensive performance to achieve next revolutionary technology node. 
Phase-change random-access memory (PCRAM) is one of the most mature emerging non-volatile memory (eNVM) technology, which is expected to achieve a processing-inmemory architecture applicable to big data ${ }^{1}$, artificial intelligence ${ }^{2,3}$ and other fields ${ }^{4}$. Being the focus of research and development for the chip industry and academia, PCRAM utilizes the ultrafast transition $(\sim \mathrm{ns})$ between the highresistance amorphous and low-resistance crystalline phases of phase-change materials (PCMs) to store information. ${ }^{5}$ It has the advantages of non-volatility, three-dimensional integratability, multi-bit memory, good scalability and compatibility with CMOS process. ${ }^{6}$ To fabricate commercial PCRAM, writing speed and data retention are two key performances, which are $\sim 50 \mathrm{~ns}$ and $80{ }^{\circ} \mathrm{C}$ for commercially used $\mathrm{Ge}_{2} \mathrm{Sb}_{2} \mathrm{Te}_{5}$ (GST), respectively. ${ }^{7}$ But they are still currently fall short of the expectations from an ideal PCM. Alloy design or doping impurity is a practical strategy to improve them. It is always believed that accelerating the speed is incompatible with the improvement of data retention, seeing the review ${ }^{8}$. However, many doped systems of lattice replacement do not present such contradictory ${ }^{6,9,10,11}$, albeit some systems with mismatched dopant meeting the experience $^{12,13}$. Therefore, doping impurity with lattice replacement is an effective approach to solve the contradictory.

Nevertheless, direct atomic detail of how dopant with lattice replacement influences the kinetic pathway is still

(a)

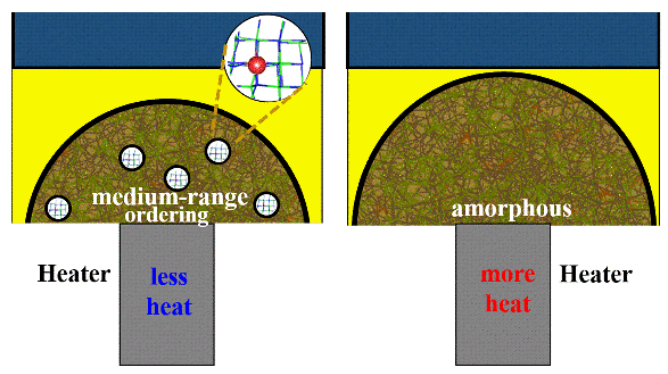

Hierarchical Melt Complete Melt

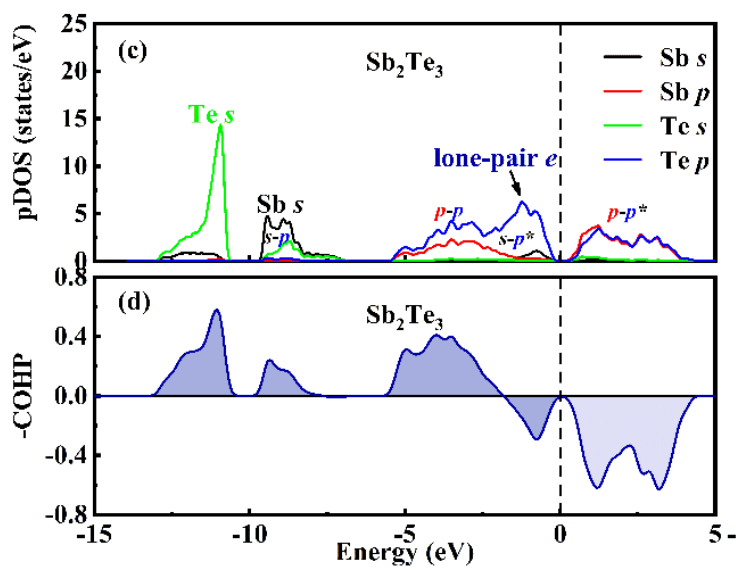

missing. Recently, Sc-Sb-Te (SST) material was reported to offer a record-breaking speed of $0.7 \mathrm{~ns}$. $^{9}$ It is explained by the octahedral local motif around $\mathrm{Sc}$ dopant to reduce the stochasticity of nucleation, but of no more kinetic details. Moreover, although Y element has less octahedral-like local pattern in amorphous ${ }^{9,}{ }^{14}, \mathrm{Y}-\mathrm{Sb}-\mathrm{Te}$ (YST) material still can achieve one order of magnitude faster speed than GST. ${ }^{11}$ While we can enable the slow GST crystallization process via ab initio molecular dynamics (AIMD) simulation ${ }^{15,16}$, not any indication of nucleation was observed in YST by a long-time AIMD simulation. In other doped systems, their fast crystallization trajectories were also hard to recurrence by simulations. ${ }^{6,9,10,11}$ Thus, a gap between the speed predicted by simulation and experiment result should be bridged.

To fill the gap, we should understand the practical nucleation process at first. In liquids, cooperative movement $(\mathrm{CM})$ as well as the similar concept of cooperatively rearranging region is a general feature as particle moves, which is manifested by both simulations ${ }^{17}, 18,19,20,21$ and experiments $^{22,23}$. By monitoring the crystallization process, the $\mathrm{CM}$ process is necessary and monitored in a good metallic glass-former $\mathrm{CuZr}$, where more crystal-like rejuvenated disorder states should be encountered before nucleation. ${ }^{24} \mathrm{On}$ account of the formation of strong bonds in the doped PCMs, the isolated dopants, even with octahedral motifs, make the CMs more difficult and impedes the critical nucleation of
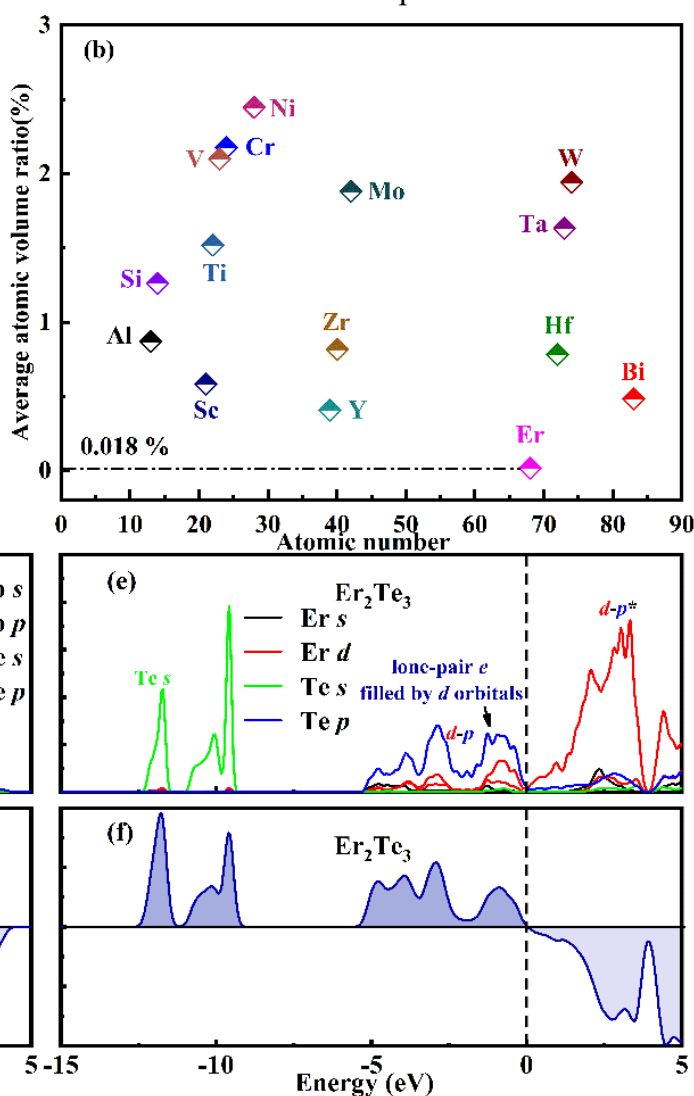

Fig. 1 Alloy design. (a), the schematic diagrams of device cells with different amorphous states as different power is provided. The left part has many medium-range ordering regions as less heat is provided, while the right part is a fully disordered amorphous state as more heat is provided. (b), the lattice mismatch between metal tellurides and $\mathrm{Sb}_{2} \mathrm{Te}_{3}$, where Er has the least mismatch of volume change rate of $0.018 \%$. (c)-(f), evidences of orbital interactions by calculating the pDOSs and COHP of $\mathrm{Sb}_{2} \mathrm{Te}_{3} \mathrm{and} \mathrm{Er}_{2} \mathrm{Te}_{3}$. (c), (e), The pDOSs for various orbitals. (d), (f), the -COHP curves for $\mathrm{Sb}_{2} \mathrm{Te}_{3}$ and $\mathrm{Er}_{2} \mathrm{Te}_{3}$, respectively. 
many crystal-like atoms connected. ${ }^{15}$ Although reference has manifested that it is stable, compared with other regions, for the artificial embryo of a heterogeneous crystallite during a short-time melting simulation ${ }^{25}$, it is unpractical to sow them using the sputtering technique and the embryo can melt as it undergoes enough time at high temperature. Here, we propose a hierarchical melt concept to produce a medium-range crystal-like region acting as a precursor, which is stabilized by several neighbor foreign atoms as less heat is provided, similar to the intention of low-voltage-incubation operation to form prestructural ordering ${ }^{7,26}$.

On the other hand, why dopants stabilize amorphous still puzzles us, unknown of which challenges the choose of dopant to improve data retention. Generally, a criterion is used by choosing metals with high cohesive energy or high melting point. ${ }^{9}$ While some elements, such as $\mathrm{Al}^{27}, \mathrm{Ga}^{28}, \mathrm{In}^{29}$, and $\mathrm{Sn}^{30}$, present low cohesive energy or low melting point, they, to our surprise, can stabilize the amorphous well. This gives us a hint that it probably exists a deeper reason. Noteworthily, chalcogenide elements have electron-redundant nature or lone-pair electrons ${ }^{31,32,33}$, which can be filled by the empty orbitals of neighbor atoms to form coordinate bond ${ }^{32,33}$. Thus, forming extra coordinate bonds to chalcogenide elements provides a way to stabilize amorphous.

Here, we utilize hierarchical melt and coordinate bond concepts to design better comprehensive performance of PCMs. The hierarchical melt can be achieved by controlling the operation process of providing less heat (short pulse), whose schematic diagram is shown in Fig. 1a. The special electrical operation process of high-speed test is shown in Fig. S1. In order to choose dopants with lattice replacement, we
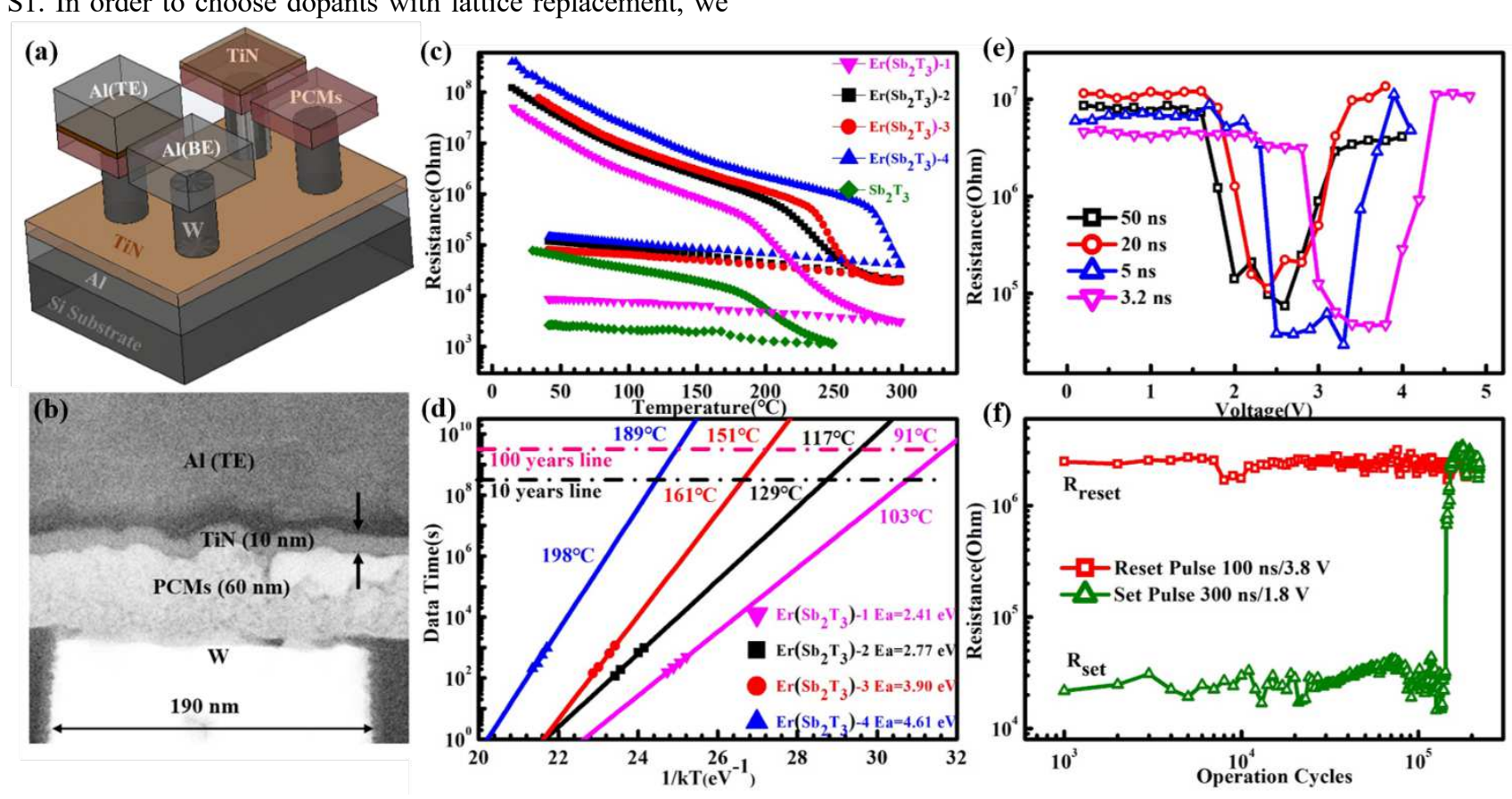

Fig. 2 Device performances. (a), schematic diagram of T-type phase change memory cell. (b), the TEM image of the cross-section of the memory cell. (c), temperature dependence of the resistance for $\mathrm{Sb}_{2} \mathrm{Te}_{3}$ and $\mathrm{Er}_{x} \mathrm{Sb}_{2} \mathrm{Te}_{3}$ films at the same heating rate of 10 $\mathrm{K} \cdot \mathrm{min}^{-1}$. (d), the 10-year (or 100-year) data retention temperature and activation energy of crystallization are deduced from the extrapolated fitted lines based on the failure time versus reciprocal temperature. (e), the switching property characterized by the SET-RESET windows for $\mathrm{Er}_{0.52} \mathrm{Sb}_{2} \mathrm{Te}_{3}$. (f), the operation cycles for $\mathrm{Er}_{0.52} \mathrm{Sb}_{2} \mathrm{Te}_{3}$. focus on metals, whose degree of mismatch with parent $\mathrm{Sb}_{2} \mathrm{Te}_{3}$ is summarized in Fig. 1b. It shows that erbium (Er) has the least mismatch volume change rate, which has stable cubic empty $d$ orbitals of Er can be filled by lone-pair electrons of Te atoms, manifested by partial density of states (pDOS) and crystal orbital Hamilton populations (COHP) shown in Fig. 1c-f. In the following, the excellent comprehensive performance of Er-doped $\mathrm{Sb}_{2} \mathrm{Te}_{3}$ is exhibited and explained.

Device performance.

Our investigation starts by experimentally testing the device performance of Er-doped $\mathrm{Sb}_{2} \mathrm{Te}_{3}$, as shown in Fig. 2. To evaluate data retention, we utilize the resistance-temperature or -time $(R-T$ or $R$ - $t)$ curves, as shown in Fig $2 \mathrm{c}-\mathrm{d}$. The sharp drop of $R-T$ curves is defined as crystallization temperature $\left(T_{\mathrm{c}}\right)$, and the tested $T_{\mathrm{c}}$ values of four compositions, $\mathrm{Er}_{x} \mathrm{Sb}_{2} \mathrm{Te}_{3}$ $(x=0.31,0.41,0.52,0.76)$, are $203^{\circ} \mathrm{C}, 235^{\circ} \mathrm{C}, 256^{\circ} \mathrm{C}$, and $289^{\circ} \mathrm{C}$, respectively. It demonstrates that $\mathrm{Er}$ can improve amorphous stability significantly, explained in the following. It is noted that the resistance is increased for both amorphous and crystalline phases after doping Er, which is helpful to $129^{\circ} \mathrm{C}\left(117^{\circ} \mathrm{C}\right), 161^{\circ} \mathrm{C}\left(151^{\circ} \mathrm{C}\right)$, and $198^{\circ} \mathrm{C}\left(189^{\circ} \mathrm{C}\right)$, respectively. They are much higher than the commonly used PCMs, such as GST $\left(\sim 80^{\circ} \mathrm{C}\right)^{35}$, SST $\left(\sim 85^{\circ} \mathrm{C}\right)^{9}$, even nonmetaldoped $\mathrm{C}-\mathrm{GST}^{36}$ and $\mathrm{N}-\mathrm{GST}^{37}$, which meets the temperature requirement of most non-volatile applications ${ }^{28}$. ErTe and $\mathrm{Er}_{2} \mathrm{Te}_{3}$ phases in the phase diagram. Moreover, the 
By comparing the device performance of four components (Fig. S2), $\mathrm{Er}_{0.52} \mathrm{Sb}_{2} \mathrm{Te}_{3}$ (EST) has better comprehensive performance and is chosen for the following electrical tests using a T-shape cell, whose schematic diagram and crosssection are shown in Fig. 2a-b. Figure 2e shows the SETRESET windows using the resistance-voltage (R-V) curves, whose high/low resistance ratio $\left(R_{\text {reset }} / R_{\text {set }}\right)$ is about two orders of magnitude and meet the requirement of ON/OFF ratio used in PCRAM. It is noted that EST has an operating speed of $3.2 \mathrm{~ns}$, which is faster than most PCMs reported ${ }^{10,11}$, albeit still slower than SST. Its SET $\left(V_{\text {set }}\right)$ and RESET ( $\left.V_{\text {RESET }}\right)$ voltages are $3.2 \mathrm{~V}$ and $4.4 \mathrm{~V}$, respectively, which are lower than $\mathrm{GST}^{9}$ and is responsible for its low-power consumption. It is estimated by the equation ${ }^{38}, Q_{t}=\left(V_{\text {reset }}^{2} / R_{\text {set }}\right) \times t_{\text {reset }}+$ $\left(V_{\text {set }}^{2} / R_{\text {reset }}\right) \times t_{\text {set }}$, where $Q_{\mathrm{t}}$ and $t$ are required energy and operation time, respectively, and the second term is ignored due to the large $R_{\text {reset }} / R_{\text {set }}$ ratio. According to the operation window, the required energy is estimated $1.27 \mathrm{pJ}$ for EST, which is much lower than GST $(901.8 \mathrm{pJ})^{38}$ and $\mathrm{Sb}_{2} \mathrm{Te}_{3}(31.9$ $\mathrm{pJ})^{9}$, even smaller than the reported least one of YST $\left(1.3 \mathrm{pJ}^{11}\right)$. The physical reason may originate from the grain refinement, discussed in Fig. S3 and Fig. S4. Figure 2f shows the tested endurance over $10^{5}$ cycles by imposing two appropriate SET and RESET voltage pulses alternately. Thus, our designed EST has great potential for the storage-class memory applications. In the following, we focus on (i) characterizing the occupied positions of Er dopant in the experiment, and (ii) theoretically uncovering the detailed reasons why Er dopant stabilizes the amorphous and accelerates the crystallization.

Direct atomic evidence of Er replacing cationic positions. From the previous mismatch analysis, it predicts that the least mismatch Er prefers replacing lattice position, due to the least strain energy, which can avoid the separation of impurity phase. From the calculations, Er locating at the cationic position has the substitution energy of $-1.92 \mathrm{eV}$ and is $\sim 0.5 \mathrm{eV}$ lower than in the anionic position. The details are shown in S5. Figure 3 provides the experimental evidences by showing the atomic arrangement and elemental distribution in the crystalline phase of EST using in situ transmission electron microscope (TEM) measurement in a [110] oriented plane at $280^{\circ} \mathrm{C}$. Figure 3 a shows the bright field (BF) STEM image. A vacancy ordering layer (VOL) is found, but not sharing two Te-terminated boundaries for forming a vdW gap. Figure 3e shows the intensity profile of the marked rectangle region in Fig. 3a, the distance of two Te planes is about $\sim 4.4 \AA$, which is consistent with the reported value ${ }^{39}, 4.1 \sim 4.5 \AA$. It illustrates that EST is still a cubic phase at $280^{\circ} \mathrm{C}$, whose structure difference compared with hexagonal phase is shown in Fig. S6. It should be mentioned that the cation intensity near the VOL, marked by star, has similar value with its neighbor anion position, which indicates that Er with large atomic number has high content in this special position.

Figures $3 b-d$ identify the distribution of three elements via the atom-resolved energy-dispersive X-ray spectroscopy
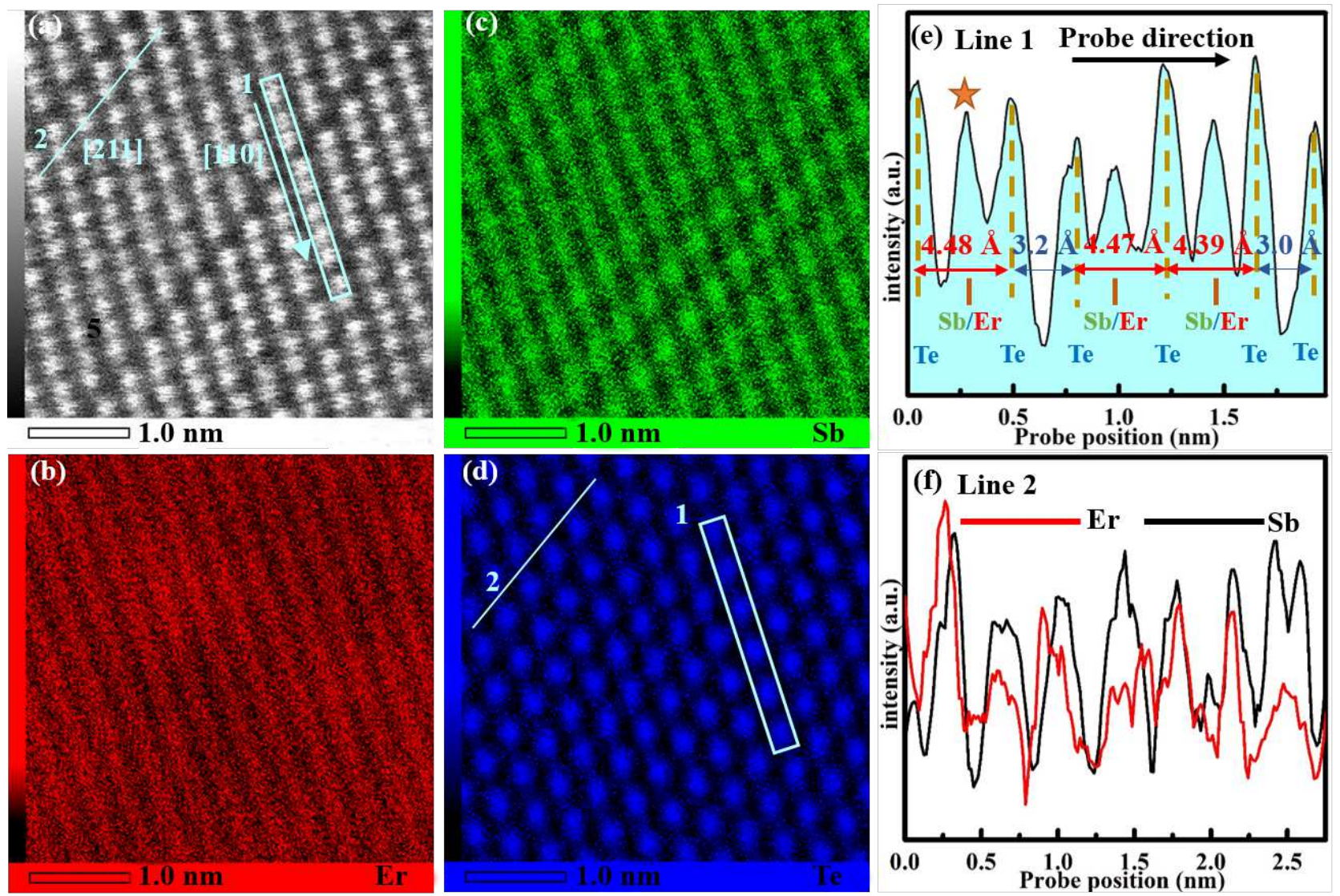

Fig. 3 Atomic structure of cubic EST phase at $280^{\circ} \mathrm{C}$. (a), BF image. (b)-(d), the atomic resolution map images of Er (red), Sb (green) and Te (blue) atoms taken from (a). (e), the intensity profile of marked rectangle region in line 1. (f), the contrast intensity of Er and Sb along line 2. 
(EDX). We clearly resolve the distribution of Er dopant in the [211] direction, but the blurry resolution in the [110] direction, as shown in Fig. 3b, which may attribute to the complicated shape of Er $d$ orbitals. Figure 3c shows the distribution of $\mathrm{Sb}$ atoms, whose resolution is clear in the Er-poor region. In order to verify both $\mathrm{Er}$ and $\mathrm{Sb}$ atoms in the Er-enrich region, we check the intensity of Er and Sb along the cyan line 2 in Fig. 3a, as shown in Fig. 3f. The coupled intensity peaks of Er and $\mathrm{Sb}$ demonstrates that they are both in the cationic position. In the XPS test, the blueshift of the binding energy of Te $3 d$ orbital further manifests the interaction between the Er atoms and anionic Te atoms, as shown in Fig. S7. Therefore, we can make a conclusion that Er locates at cationic positions.

Stabilizing anionic Te atoms by forming coordinate bonds. Subsequently, we uncover the reason why dopant stabilizes the amorphous by the chemical bonding analysis. Figure $4 a-b$ show the pDOS and COHP in the amorphous. Similar to the scenario in the crystalline $\mathrm{Sb}_{2} \mathrm{Te}_{3}$ phase shown in Fig. 1, all bonding $p_{\mathrm{Sb}}-p_{\mathrm{Te}}$ orbitals below Fermi level and all antibonding $p_{\mathrm{Sb}}-p_{\mathrm{Te}}$ * orbitals above Fermi level. The $s$ orbitals of both $\mathrm{Sb}$ and $\mathrm{Te}$ atoms mainly locate at deep energy levels, albeit a weak hybridization between the $s$ orbitals ( $\mathrm{Sb}$ ) with $p$ orbitals (Te), where the anti-bonding $s-p^{*}$ orbitals is just below Fermi level. Remarkably, we find the lone-pair electrons of Te atoms fill the empty $d$ orbitals of Er atoms, the same as the above discussion in the ErTe material, which stabilizes the amorphous of anionic Te atoms.

Figure $4 \mathrm{c}$ shows the pair correlation functions (PCFs) of $\mathrm{Er}-\mathrm{Sb}, \mathrm{Er}-\mathrm{Te}, \mathrm{Sb}-\mathrm{Sb}, \mathrm{Sb}-\mathrm{Te}$, and $\mathrm{Te}-\mathrm{Te}$ pairs in the amorphous. For the first peak, we find that the $\mathrm{Er}-\mathrm{Te}$ is the highest. To our surprise, Te-Te homopolar bonds are almost disappeared according to its weak first peak, against the fact of huge homopolar bonds in the common amorphous $\mathrm{PCMs}^{40}$. The disappeared $\mathrm{Te}-\mathrm{Te}$ homopolar bonds may attribute to the preferred Er-Te interaction. The observed $\mathrm{Sb}-\mathrm{Sb}$ homopolar bonds is because of weak $\mathrm{Er}-\mathrm{Sb}$ interaction with lower first peak. Moreover, the dynamic property, such as diffusion coefficient, can further verify the stabilized Te atoms. The mean-square displacement (MSD) is calculated for $\operatorname{Er}$ (black), $\mathrm{Sb}$ (red), and Te (green) atoms. It shows that Er has the least displacement, because of its maximum coordinate number shown in S8 and many coordinate bonds formed with Te atoms. The Te displacement is about half of Sb displacement, compared with their similar values in other GST systems ${ }^{40,41}$. Therefore, a conclusion is made that the formed coordinate bond is the essential reason of the stabilized amorphous.

On the other hand, the formed coordinate bond also exists in the crystalline phase and has a significant effect on PCM properties. It has been proved that Te atoms near vacancy traps electrons near Fermi level ${ }^{42}$, as well as lone-pair electrons near Fermi level shown in Fig. 1c-e. Thus, we can predict that Er prefers the cationic position near vacancy by sharing the empty $d$ orbitals with the neighbor lone-pair electrons of Te atoms to form coordinate bonds, which is in line with the above experiment result of high content Er near the VOL. The VOL (or Te-terminated boundary) is also stabilized at the same time. It can refine grains seriously, because our recent work has proved that the extraordinarily stable Te-terminated boundary is the reason of small grain size in some PCMs. ${ }^{43}$ The increased number of grain boundary will hinder the
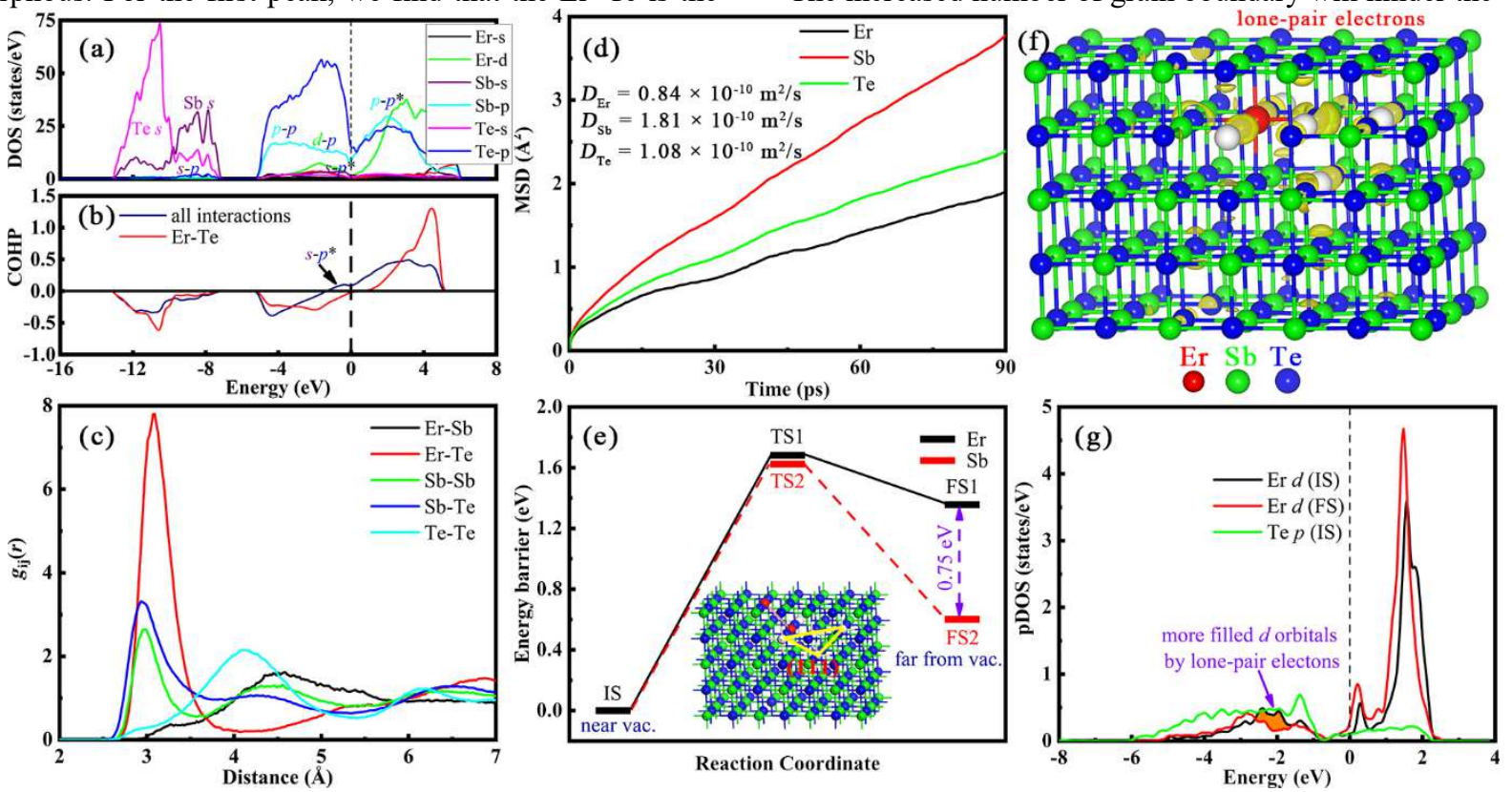

Fig. 4 Evidences of stabilized Te atoms in both amorphous (a)-(d) and crystalline phases (e)-(g) by filling the empty $d$ orbitals of Er atoms using the lone-pair electrons. (a), the pDOS of Er, Sb, and Te atoms with various orbitals. (b), the COHP of average values of all interactions and Er-Te interaction, respectively. (c), the PCFs of Er-Sb (black), Er-Te (red), $\mathrm{Sb}-\mathrm{Sb}$ (green), $\mathrm{Sb}-\mathrm{Te}$ (blue), and Te-Te (cyan) pairs. (d), the MSDs for Er, Sb, and Te atoms. (e), the energetic profile of diffusion barrier for Er (black) or Sb (red and dash), where a model with a four-vacancy-aggregated cluster in the (111) plane is shown in the inner graph. (f), the partial charge in the DOS region of $[-3,0] \mathrm{eV}$ in the IS structure, where 3D isosurface value is set as $0.2 \mathrm{e} \AA^{-3}$. (g), the partial DOS of Er $d$ (black) and Te $p$ (green) orbitals in the IS structure, while the Er $d$ (red) orbital in the FS structure also shown for comparison. It is noted that the pDOS of Te $p$ orbitals is divided by Te number (48 herein). 
charge transport property ${ }^{43,44,45}$ and scatters phonons ${ }^{46}$, which boost heat efficiency and more evidences see Fig. S3 and S4.

Next, we provide the calculation evidences of the formation of coordinate bond and the stabilized Te-terminated boundary. Figure 4e shows the diffusion barrier of $\mathrm{Er}$ (black) or $\mathrm{Sb}$ (red) migrating from near the four aggregated vacancies in the (111) plane to the inner vacancy. Remarkably, the final state (FS) of Er has the energy of $\sim 1.4 \mathrm{eV}$ higher than the initial state (IS). It is still $0.75 \mathrm{eV}$ higher than that the FS of Sb migration, both of which have similar migration barrier. The much unstable FS state illustrates that VOL traps Er movement seriously. To unravel the further reason, we compare the pDOS difference between the IS and FS states, as shown in Fig. 4g. We find that more lone-pair electrons fill the $d$ orbitals of Er atoms in the IS state, because lone pair electrons prefer Te atoms near vacancies, manifested by the partial charge distribution in the region of $[-3,0] \mathrm{eV}$, as shown in Fig. $4 \mathrm{f}$.

Hierarchical melt tuned crystallization.

Finally, to uncover how Er dopant influences the crystallization kinetics, we monitor the process without any embeded seeds, where a low concentrate model, $\mathrm{Er}_{4} \mathrm{Sb}_{72} \mathrm{Te}_{108}$, is used. Firstly, we carry out a simulation of a complete-melt model using the common melt-quench method. The sample melts fully at $3000 \mathrm{~K}$ for $120 \mathrm{ps}$, and then quenches to $600 \mathrm{~K}$ for crystallization annealing. Figure 5a shows the structural evolution (red) of EST characterized by $q_{6}{ }^{\text {dot }}$ parameter during the crystallizaiton, which monitors the ordering of local environment, defined in the method. Figures 5b-f are five snapshots, where we only connect the bonds among crystallike atoms defined as $q_{6}{ }^{\text {dot }}>0.45$ as well as its neighbor atoms. At the beginning in Fig. 5b, four Er atoms are randomly distributed in the system. At $750 \mathrm{ps}, \mathrm{a} \mathrm{Sb}_{7} \mathrm{Te}_{11}$ nucleus is shown in Fig. 5c. Near the nucleus, a Er atom without octahedral pattern, marked by $\mathrm{Er}_{\mathrm{I}}$, is monitored. As the nucleus grows, the local environment of $\mathrm{Er}_{\mathrm{I}}$ atom changes to octahedron and becomes one part of the small crystallite, as shown in Fig. 5f. It is noted that a Er atom with octahedral environment, marked by $\mathrm{Er}_{\text {II }}$, is found far from the nucleus at $750 \mathrm{ps}$, while the $\mathrm{Er}_{\text {II }}$ motif becomes non-octahedron at 780 ps as the nucleus grows up to nearby it. Interestingly, the local
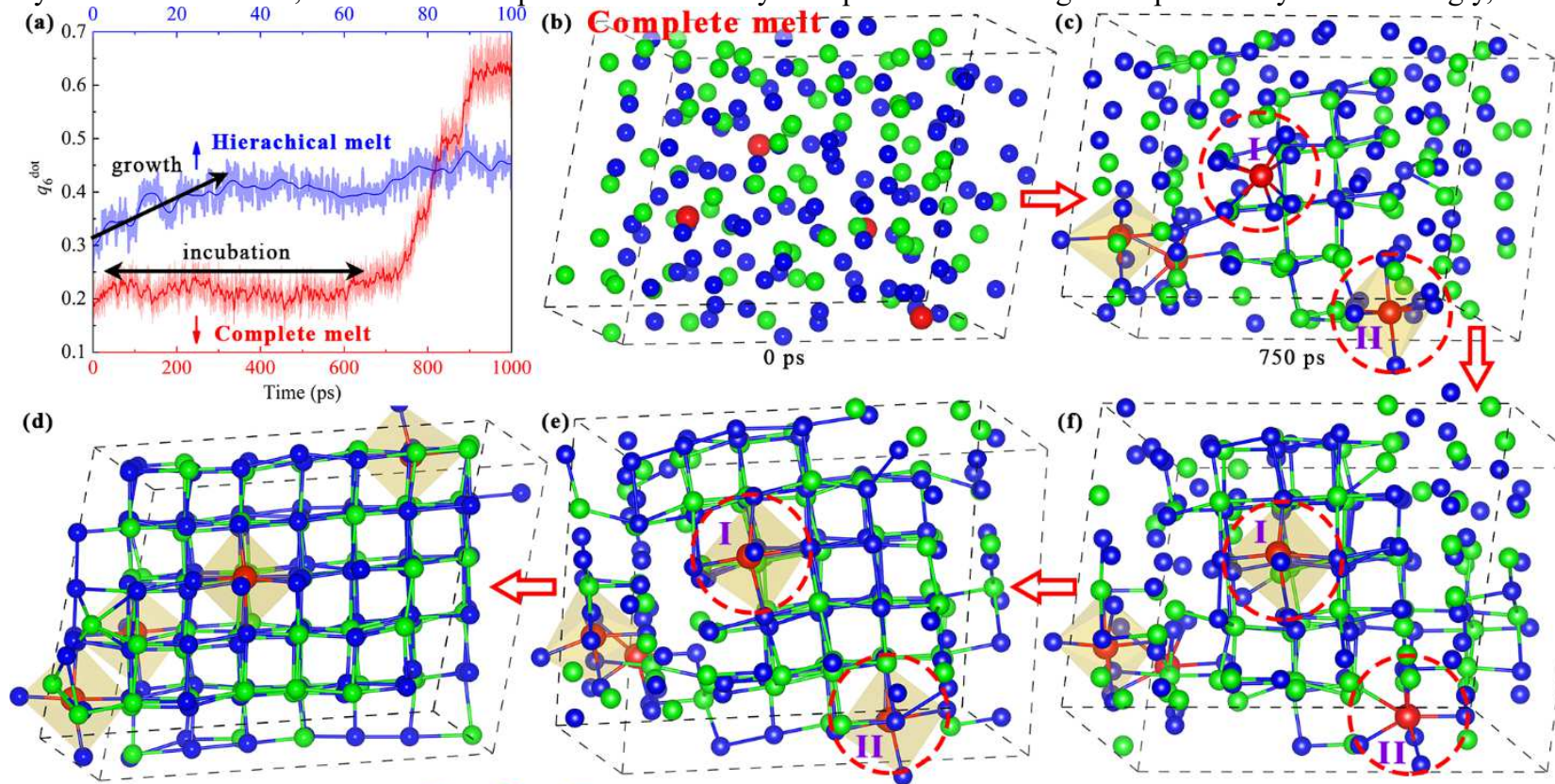

$900 \mathrm{ps}$
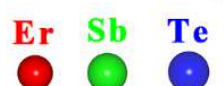

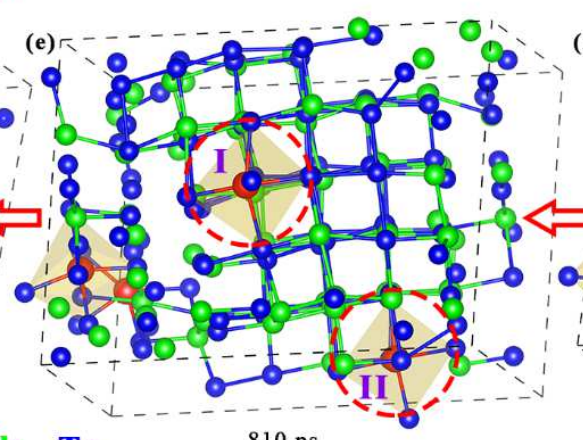

$810 \mathrm{ps}$

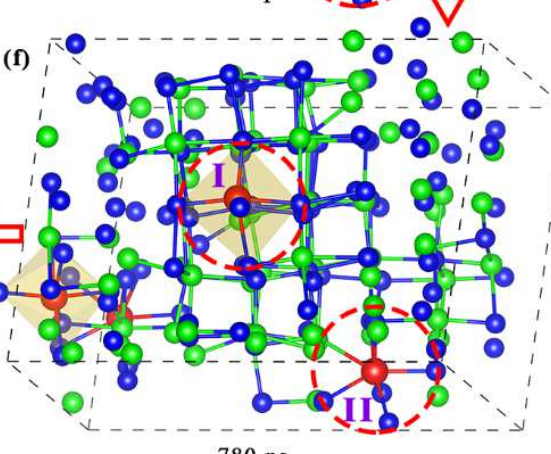

$780 \mathrm{ps}$

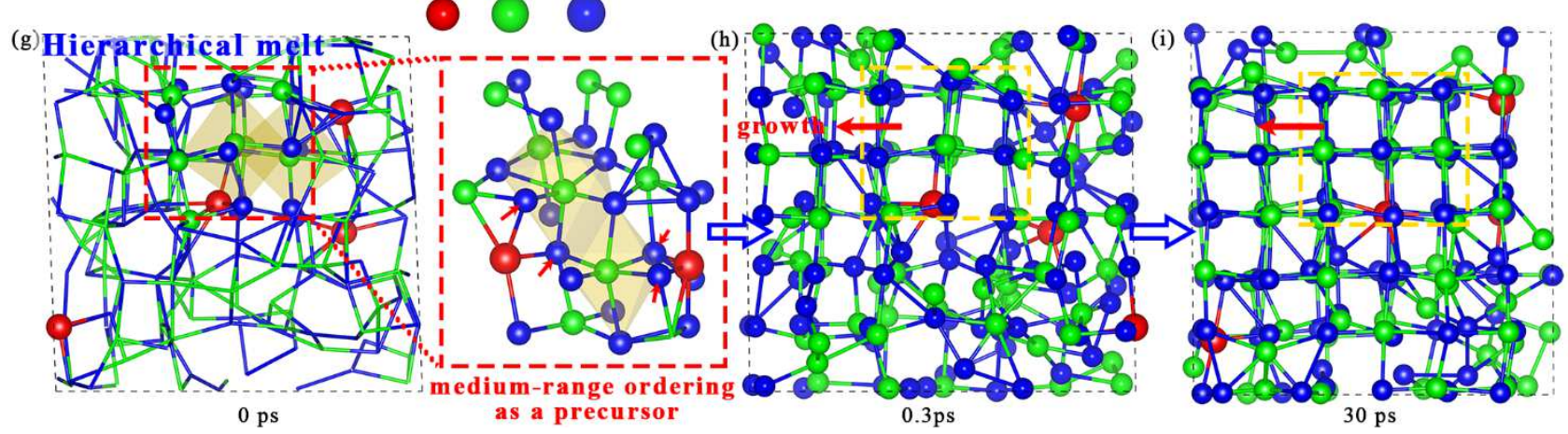

Fig. 5 Hierarchical melt tuned crystallization. (a), the evolution of $q_{6}{ }^{\text {dot }}$ value during the crystallization for the complete melt (red) and hierarchical melt (blue) models. In the complete model, (b)-(f) show the snapshots at 0 ps, 750 ps, 780 ps, 810 ps, and 900 ps, respectively, where the octahedral environment around Er atoms is characterized by orange octahedral cages. The crystal-like atoms and their neighbor atoms are connected by bonds with the cutoff of $3.6 \AA$. The red circles in (c)-(e) emphasize the alterable Er local environments. In the hierarchical melt model, (g)-(i) show the snapshots at 0ps, 0.3ps, and 30ps, respectively. 
pattern of $\mathrm{Er}_{\text {II }}$ finally changes to octahedron again at $810 \mathrm{ps}$ and it integrates into the crystallite. Therefore, the alterable local motifs of Er atoms manifest the necessary of a series of CMs during the crystallization. Finally, all Er dopants locat at the cationic positions, as shown in Fig. 5d, agreeing with above theoretical prediction and experimental observation.

However, the incubation time in the complete melt model is much longer than the reference simulation result of $\mathrm{GST}^{41}$, which seemingly conflicts with our chip test of much faster speed than GST. In the complete melt model, all atoms are fully disordered. The stabilized Te atoms by Er slow down the CMs as well as the formation of critical nucleation. It probably exists another pathway to achieve the fast speed tested in the experiment. It is noted that, in the actual highspeed test, it is hard to obtain the high speed by directly annealing the as-deposited amorphous state (complete model). In fact, a much shorter pulse (or less heat) should be providedto melt the crystalline state for the high-speed chip test, which achieves a hierarchical melt discussed at the very beginning.

In the following, we utilize the hierarchical melt model to bridge the gap between the theoretical prediction and experimental result. Based on the fact of Er preferring the canionic positions near VOL revealed above, we construct a crystalline model that two Er atoms are close to VOLs, as shown in Fig. S9. The model is melt at $1500 \mathrm{~K}$. At $1.5 \mathrm{ps}$, we find a medium-range region that is crystal-like and has two regular Sb-centre octahedrons, which is stabilized by the two Er atoms near the VOLs, as shown in Fig. $5 \mathrm{~g}$. The enlarged local structure shows that Er atoms stabilize four Te atoms constituting two octahedrons. Annealing at $600 \mathrm{~K}$, this medium-range region gradually grows up without obvious incubation, which is extremely different from the complete model. At 30ps, the nucleus grows to a big one, ableit some region still disorder because of the kinetic constraint around the relative unstable Er atoms that slows down CMs. Finally, the crystallization is accomplished after a serias of CMs. The hierarchical melt model is also suited to understand the processes of accelerated crystallization kinetics influenced by other substituted dopants, such as $\mathrm{Sc}^{9}$ and $\mathrm{Y}^{11}$.

In summary, based on the newly proposed rules of hierarchical melt and coordinate bond, we design a new $\mathrm{Er}_{0.52} \mathrm{Sb}_{2} \mathrm{Te}_{3}$ material, whose excellent device performance surpass most PCMs have been reported. It has a great potential for future application in storage-class memory: $3.2 \mathrm{~ns}$ operation speed, $161{ }^{\circ} \mathrm{C}$ data retention, more than $10^{5}$ endurance, $1.29 \mathrm{pJ}$ power consumption, and $0.41 \%$ densitychange rate. The two rules solve the contradiction of writing speed with data retention, and provide a general way to design storage-class memory with comprehensive performance.

\section{Methods}

Characterizing Film Samples: Radio-frequency (RF) magnetron cosputtering method with Er and $\mathrm{Sb}_{2} \mathrm{Te}_{3}$ targets is used to deposit the $\mathrm{Sb}_{2} \mathrm{Te}_{3}$ films and Er doped $\mathrm{Sb}_{2} \mathrm{Te}_{3}$ films. The components of the designed films were identified by sputtering power and measured by energy dispersive spectroscopy (EDX). Films with a thickness of $200 \mathrm{~nm}$ were deposited on $\mathrm{SiO}_{2} / \mathrm{Si}(100)$ substrates for resistance-temperature (R-T) and X-ray diffraction (XRD) tests. The resistance by a function of the temperature (R-T) was performed in a vacuum chamber with the heating rate of $10{ }^{\circ} \mathrm{C} \cdot \mathrm{min}^{-1}$, and isothermal change in resistance with elevated temperature was recorded to estimate the 10 -year data retention. The $\mathrm{X}$ ray reflectivity (XRR) experiment (Burker D8 Discover) was employed to test the density change before and after the crystallization of $\sim 40 \mathrm{~nm}$ thickness films. XRD was adopted to characterize the lattice information of films. X-ray photoelectron spectroscopy (XPS) experiment was used to evaluate the bonding situation. Then the microstructure of these samples was studied by JEM-ARM 300F Transmission Electron Microscope (TEM) and High-resolution transmission electron microscope (HRTEM), as well as in selected area diffraction (SAED) mode.

Fabrication PCRAM Devices: T-shaped phase-change random-access memory (PCRAM) devices with tungsten plug bottom electrode contact $(\mathrm{BEC}$, diameter $=190 \mathrm{~nm})$ are fabricated using the $0.13 \mu \mathrm{m}$ node complementary metal-oxide semiconductor technology. The $60 \mathrm{~nm}$-thick phase change material and $10 \mathrm{~nm}$-thick TiN as an adhesion layer are deposited using the sputtering method over the tungsten electrode, then $300 \mathrm{~nm}$-thick $\mathrm{Al}$ is deposited by using UMS500P Electron Beam Evaporation to form top electrode. The current-voltage (I-V), resistance-voltage $(\mathrm{R}-\mathrm{V})$, and endurance tests are carried out using a Tektronix AWG-4012 and 5002B arbitrary waveform generator and a Keithley-2400 meter parameter analyzer. The cell resistance after applying voltage pulses was recorded at a constant read voltage of $0.1 \mathrm{~V}$. First-Principle Calculations: First-principle calculations are carried out using VASP package. ${ }^{47}$ The Kohn-Sham equations are solved using the projector augmented wave (PAW) method ${ }^{48}$ and projector augmented wave (PAW) method ${ }^{48}$ and generalized gradient approximation (GGA) functional ${ }^{51}$ with the kinetic energy cutoff of $388 \mathrm{eV}$. Molecular dynamics is carried out to study the effect of dopant on crystallization kinetics at $600 \mathrm{~K}$, where we use a time step of 3 fs with Parrinello-Rahman barostat and Langevin thermostat. The degree of crystallinity is judged by $q_{6}{ }^{\mathrm{dot}}$ parameter, defined in S10, which is a modified Steinhardt order parameter to describe the similarity of one atom with its surrounding atoms.

COHP Analysis: High-precision calculations are carried out using VASP package, and then implement the pCOHP bonding analyses using LOBSTER setup ${ }^{52}$

Energy barrier calculation. The energy barrier of boundary migration is calculated by the novel Stochastic Surface Walking (SSW) method ${ }^{53}$ via smooth surface walking along softened random directions. We utilize recently developed SSW reaction pathway method to sample the grainboundary-migration pathways. ${ }^{54}$ The explicit transition states (TSs) of the pathways are located by the variable-cell double-ended surface walking (VC-DESW) method. ${ }^{55}$ The calculation details are consistent with our previous work. ${ }^{56}$

\section{Acknowledgements}

Supported by the National Key Research and Development Program of China (2017YFA0206101, 2017YFB0701703, 2017YFA0206104,2018YFB0407500,SQ2017YFGX020134), "Strategic Priority Research Program" of the Chinese Academy of Sciences (XDA09020402), National Integrate Circuit Research Program of China (2009ZX02023-003), National Natural Science Foundation of China (61904189, 61874129, 61874178, 61504157, 61622408), Science and Technology Council of Shanghai (17DZ2291300, 18DZ2272800).

\section{Additional information}

The authors declare no competing financial interests.

\section{References}

1. Big data needs a hardware revolution [editorial]. Nature 554, 145 (2018).

2. Feldmann J, Youngblood N, Wright CD, Bhaskaran H, Pernice WHP. All-optical spiking neurosynaptic networks with self-learning capabilities. Nature 569, 208-214 (2019).

3. Zhang W, Mazzarello R, Wuttig M, Ma E. Designing crystallization in phase-change materials for universal memory and neuro-inspired computing. Nat Rev Mater 4, 150-168 (2019).

4. Gu M, Zhang Q, Lamon S. Nanomaterials for optical data storage. Nat Rev Mater 1, 12 (2016).

5. Matthias W, Noboru Y. Phase-change materials for rewriteable data storage. Nat Mater 6, 824 (2007).

6. Song ZT, et al. High Endurance Phase Change Memory Chip Implemented based on Carbon-doped Ge2Sb2Te5 in $40 \mathrm{~nm}$ Node for Embedded Application. In: IEEE Int. Electron Devices Meet.) (2018).

7. Loke D, et al. Breaking the Speed Limits of Phase-Change Memory. Science 336, 1566-1569 (2012).

8. Zambelli C, Navarro G, Sousa V, Prejbeanu IL, Perniola L. Phase Change and Magnetic Memories for Solid-State Drive Applications. Proc IEEE 105, 1790-1811 (2017).

9. Rao F, et al. Reducing the stochasticity of crystal nucleation to enable subnanosecond memory writing. Science 358, 1423-1427 (2017).

10.Zhu M, et al. One order of magnitude faster phase change at reduced power in Ti-Sb-Te. Nat commun 5, 4086-4086 (2014).

11. Liu B, et al. Y-Doped Sb2Te3 Phase-Change Materials: Toward a 
Universal Memory. ACS Appl Mater Interfaces 12, 20672-20679 (2020).

12.Darmawikarta K, Raoux S, Tchoulfian P, Li T, Abelson JR, Bishop SG. Evolution of subcritical nuclei in nitrogen-alloyed Ge2Sb2Te5. J Appl Phys 112, 124907 (2012).

13. Yao D, et al. Investigation on nitrogen-doped Ge2Sb2Te5 material for phase-change memory application. Solid-State Electron 79, 138-141 (2013).

14. Zhou Y, et al. Bonding similarities and differences between $\mathrm{Y}-\mathrm{Sb}-\mathrm{Te}$ and $\mathrm{Sc}-\mathrm{Sb}-\mathrm{Te}$ phase-change memory materials. J Mater Chem C 8, 3646-3654 (2020)

15. Lee TH, Loke D, Elliott SR. Microscopic Mechanism of DopingInduced Kinetically Constrained Crystallization in Phase-Change Materials. Adv Mater 27, 5477-5483 (2015).

16. Sosso GC, Miceli G, Caravati S, Giberti F, Behler J, Bernasconi M. Fast Crystallization of the Phase Change Compound GeTe by LargeScale Molecular Dynamics Simulations. J Phys Chem Lett 4, 42414246 (2013).

17. Donati C, Douglas JF, Kob W, Plimpton SJ, Poole PH, Glotzer SC. Stringlike Cooperative Motion in a Supercooled Liquid. Phys Rev Lett 80, 2338-2341 (1998)

18. Stevenson JD, Schmalian J, Wolynes PG. The shapes of cooperatively rearranging regions in glass-forming liquids. Nat Phys 2, 268-274 (2006)

19. Zhang Z, Yunker PJ, Habdas P, Yodh AG. Cooperative Rearrangement Regions and Dynamical Heterogeneities in Colloidal Glasses with Attractive Versus Repulsive Interactions. Phys Rev Lett 107, (2011).

20. Starr FW, Douglas JF. Modifying Fragility and Collective Motion in Polymer Melts with Nanoparticles. Phys Rev Lett 106, 115702 (2011).

21. Keys AS, Hedges LO, Garrahan JP, Glotzer SC, Chandler D. Excitations Are Localized and Relaxation Is Hierarchical in GlassForming Liquids. Phys Rev X 1, 021013 (2011).

22. Hanakata PZ, Douglas JF, Starr FW. Interfacial mobility scale determines the scale of collective motion and relaxation rate in polymer films. Nat Commun 5, 4163 (2014).

23. Hima Nagamanasa K, Gokhale S, Sood AK, Ganapathy R. Direct measurements of growing amorphous order and non-monotonic dynamic correlations in a colloidal glass-former. Nat Phys 11, 403408 (2015).

24. Song W-X, Yang Y, Wang W, Guan P. Revealing Rejuvenated Disorder States towards Crystallization in a Supercooled Metallic GlassForming Liquid. arXiv:2002.09669 (2020).

25. Ding K, Chen B, Chen Y, Wang J, Shen X, Rao F. Recipe for ultrafast and persistent phase-change memory materials. NPG Asia Mater 12, $63(2020)$

26. Lee TH, Loke D, Huang K-J, Wang W-J, Elliott SR. Tailoring Transient-Amorphous States: Towards Fast and Power-Efficient Phase-Change Memory and Neuromorphic Computing. Adv Mater 26, 7493-7498 (2014)

27. Peng C, et al. Al1.3Sb3Te material for phase change memory application. Appl Phys Lett 99, 043105 (2011).

28. Kao K-F, Lee C-M, Chen M-J, Tsai M-J, Chin T-S. Ga2Te3Sb5-A Candidate for Fast and Ultralong Retention Phase-Change Memory. Adv Mater 21, 1695-1699 (2009).

29. Saxena N, Persch C, Wuttig M, Manivannan A. Exploring ultrafast threshold switching in In3SbTe2 phase change memory devices. Sci Rep 9, 19251 (2019).

30. Bilovol V, et al. Structural, vibrational and electronic properties in the glass-crystal transition of thin films Sb70Te30 doped with Sn. J Alloys Compd 845, 156307 (2020).

31. Kastner M. Bonding Bands, Lone-Pair Bands, and Impurity States in Chalcogenide Semiconductors. Phys Rev Lett 28, 355-357 (1972).

32. Xu M, Cheng YQ, Sheng HW, Ma E. Nature of Atomic Bonding and Atomic Structure in the Phase-Change Ge2Sb2Te5 Glass. Phys Rev Lett 103, (2009).

33. Kolobov AV, Fons P, Tominaga J. Understanding Phase-Change
Memory Alloys from a Chemical Perspective. Sci Rep 5, 13698 (2015).

34. Boniardi $\mathrm{M}$ et al. Optimization metrics for Phase Change Memory (PCM) cell architectures. In: IEEE Int. Electron Devices Meet.) (2014). 35. Guo T, et al. SiC-Doped Ge2Sb2Te5 Phase-Change Material: A Candidate for High-Density Embedded Memory Application. Adv Electron Mater 4, 1800083 (2018).

36. Li T, Wu LC, Song ZT, Song SN, Rao F, Liu B. Carbon-Doped SbRich Ge-Sb-Te Phase Change Material for High Speed and High Thermal Stability Phase Change Memory Applications. Mater Sci Forum 898, 1834-1838 (2017).

37. Shelby RM, Raoux S. Crystallization dynamics of nitrogen-doped Ge_2Sb_2Te_5. J Appl Phys 105, 104902.104901-104902.104906 (2009).

38. Hatayama S, et al. Inverse Resistance Change Cr2Ge2Te6-Based PCRAM Enabling Ultralow-Energy Amorphization. ACS Appl Mater Interfaces 10, 2725-2734 (2018).

39. Zheng Y, et al. Direct observation of metastable face-centered cubic Sb2Te3 crystal. Nano Res 9, 3453-3462 (2016).

40. Song W-X, Liu Z-P, Liu L-M. Inherent Simple Cubic Lattice Being Responsible for Ultrafast Solid-Phase Change of Ge2Sb2Te5. J Phys Chem Lett 8, 2560-2564 (2017)

41. Lee TH, Elliott SR. Ab Initio Computer Simulation of the Early Stages of Crystallization: Application to Ge2Sb2Te5 Phase-Change Materials. Phys Rev Lett 107, (2011).

42. Zhang W, et al. Role of vacancies in metal-insulator transitions of crystalline phase-change materials. Nat Mater 11, 952-956 (2012).

43. Song W-X, et al. Crystallization Mechanism Tuned Phase-Change Materials: Quantum Effect on Te-Terminated Boundary. arXiv:2011.11159 (2020)

44. Arashdeep Singh Thind GL, Jordan A. Hachtel, Maria V. Morrell, Sung Beom Cho, Albina Y. Borisevich, Juan-Carlos Idrobo, Yangchuan Xing, and Rohan Mishra. Atomic Structure and Electrical Activity of Grain Boundaries and Ruddlesden-Popper Faults in Cesium Lead Bromide Perovskite. Adv Mater 31, 1805047 (2018)

45. Yazyev OV, Louie SG. Electronic transport in polycrystalline graphene. Nat Mater 9, 806-809 (2010).

46. Song W-X, et al. Improving the performance of phase-change memory by grain refinement. J Appl Phys 128, 075101 (2020).

47. Kresse G, Furthmuller J. Efficient iterative schemes for ab initio totalenergy calculations using a plane-wave basis set. Phys Rev B 54, 11169-11186 (1996).

48. Blöchl PE. Projector augmented-wave method. Phys Rev B 50, 17953 17979 (1994).

49. Perdew JP, Ernzerhof M, Burke K. Rationale for mixing exact exchange with density functional approximations. J Chem Phys $\mathbf{1 0 5}$, 9982-9985 (1996).

50. Grimme S, Antony J, Ehrlich S, Krieg H. A consistent and accurate ab initio parametrization of density functional dispersion correction (DFT-D) for the 94 elements H-Pu. J Chem Phys 132, 154104 (2010).

51. Perdew JP, Wang Y. Accurate and simple analytic representation of the electron-gas correlation energy. Phys Rev B 45, 13244-13249 (1992).

52. Maintz S, Deringer VL, Tchougréeff AL, Dronskowski R. LOBSTER: A tool to extract chemical bonding from plane-wave based DFT. $J$ Comput Chem 37, 1030-1035 (2016).

53. Shang C, Liu Z-P. Stochastic Surface Walking Method for Structure Prediction and Pathway Searching. J Chem Theory Comput 9, 18381845 (2013).

54. Guan S-H, Zhang X-J, Liu Z-P. Energy Landscape of Zirconia Phase Transitions. J Am Chem Soc 137, 8010-8013 (2015).

55.Zhang X-J, Liu Z-P. Variable-Cell Double-Ended Surface Walking Method for Fast Transition State Location of Solid Phase Transitions. J Chem Theory Comput 11, 4885-4894 (2015).

56. Song W-X, Liu Z-P, Liu LM. Inherent Simple Cubic Lattice Being Responsible for Ultrafast Solid-Phase Change of Ge2Sb2Te5. J Phys Chem Lett 8, 2560-2564 (2017). 
(a)
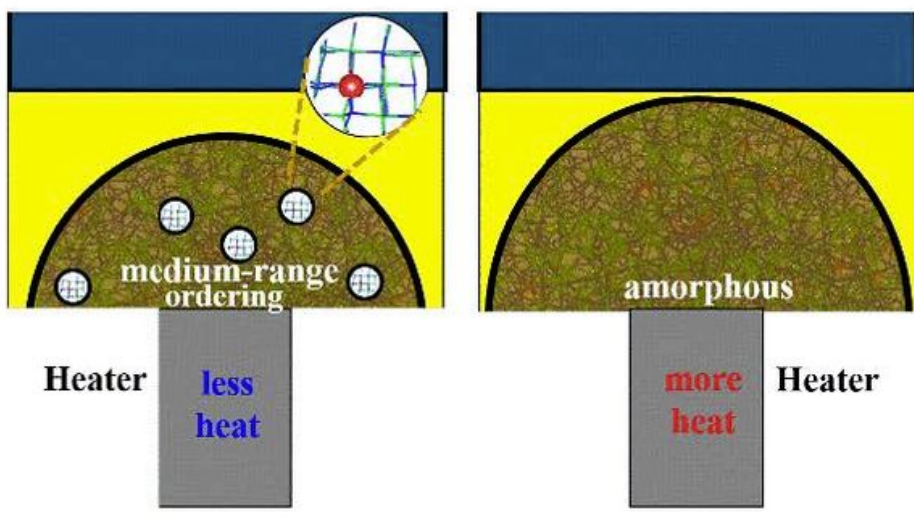

\section{Hierarchical Melt Complete Melt}

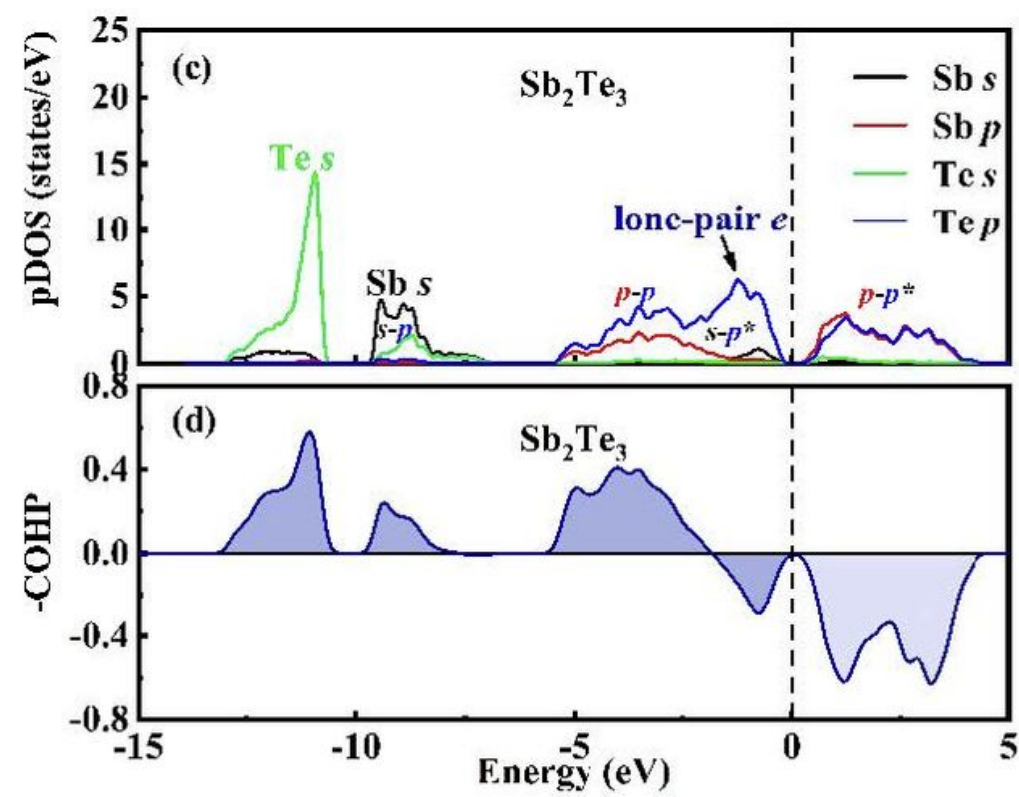

(b)

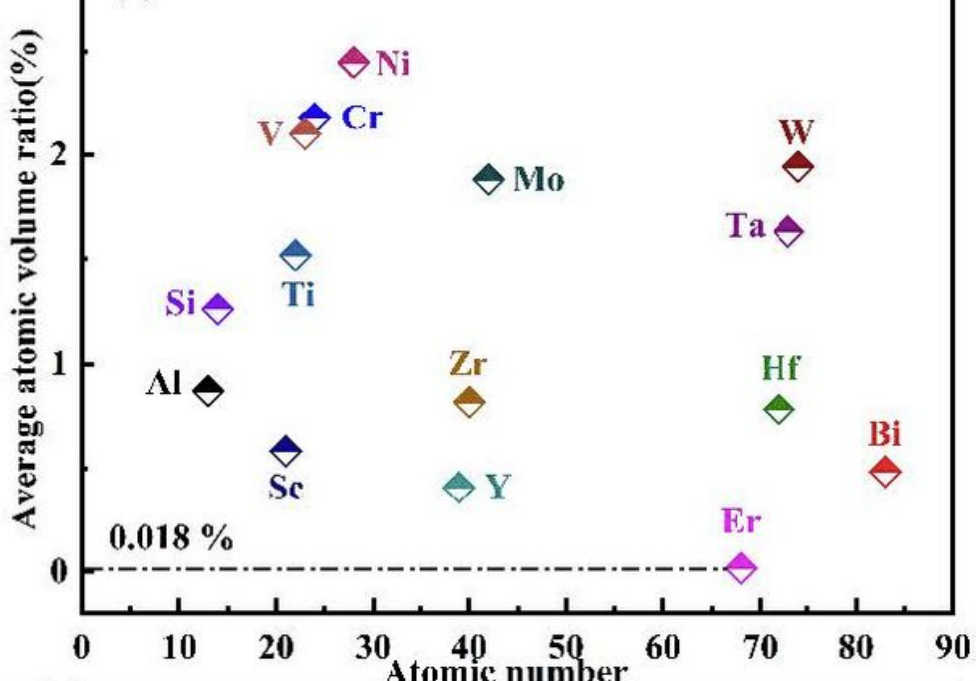

(e)

Atomic number
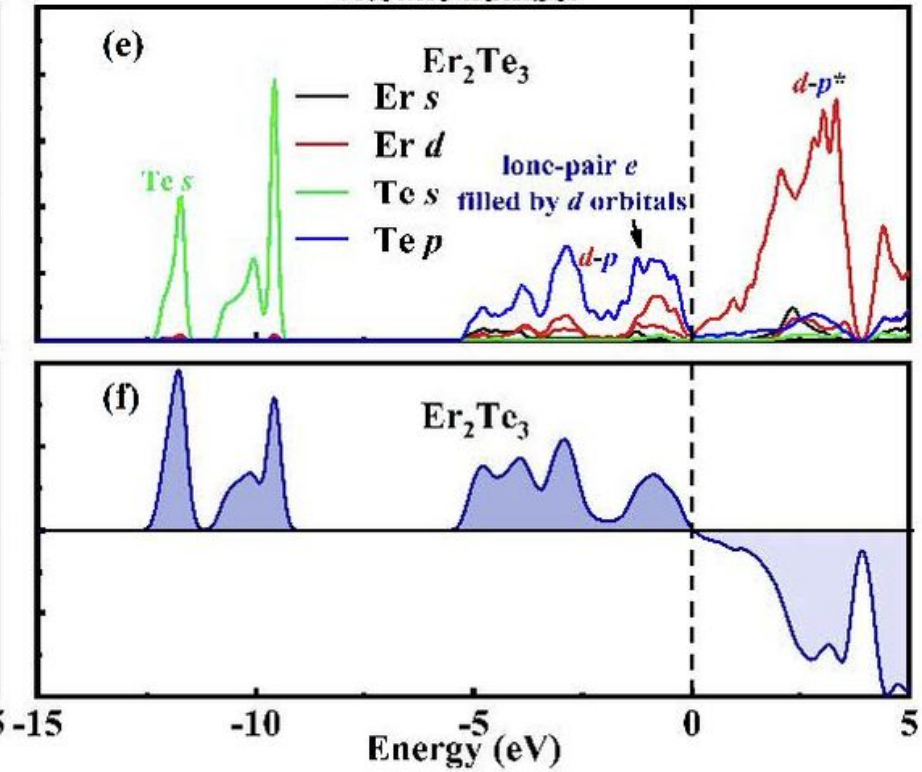

Figure 1

Alloy design. (a), the schematic diagrams of device cells with different amorphous states as different power is provided. The left part has many medium-range ordering regions as less heat is provided, while the right part is a fully disordered amorphous state as more heat is provided. (b), the lattice mismatch between metal tellurides and Sb2Te3, where Er has the least mismatch of volume change rate of $0.018 \%$.

(c)-(f), evidences of orbital interactions by calculating the pDOSs and COHP of Sb2Te3 and Er2Te3. (c),

(e), The pDOSs for various orbitals. (d), (f), the -COHP curves for Sb2Te3 and Er2Te3, respectively. 

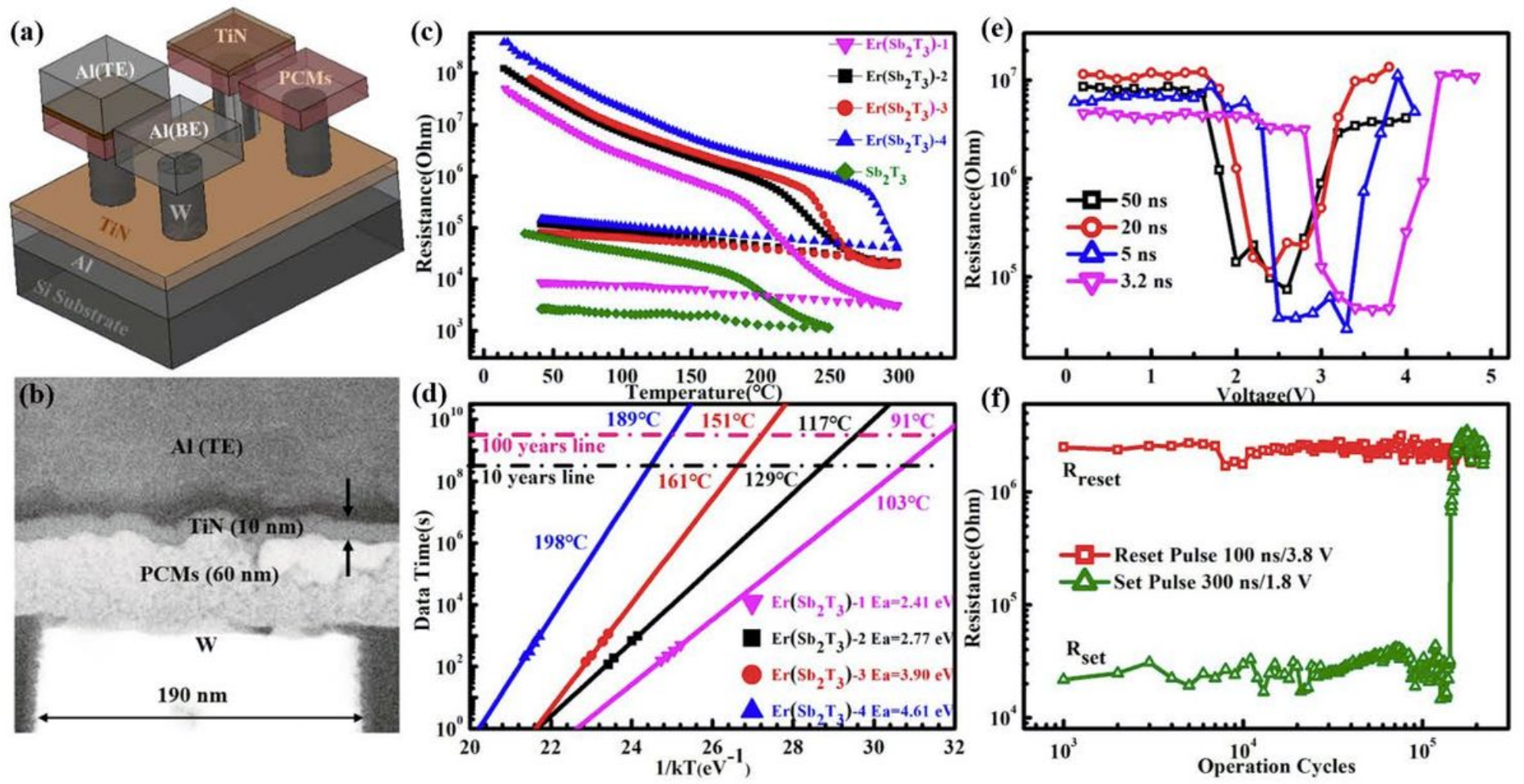

Figure 2

Device performances. (a), schematic diagram of T-type phase change memory cell. (b), the TEM image of the cross-section of the memory cell. (c), temperature dependence of the resistance for Sb2Te3 and ErxSb2Te3 films at the same heating rate of $10 \mathrm{~K} \cdot \mathrm{min}-1$. (d), the 10-year (or 100-year) data retention temperature and activation energy of crystallization are deduced from the extrapolated fitted lines based on the failure time versus reciprocal temperature. (e), the switching property characterized by the SETRESET windows for Er0.52Sb2Te3. (f), the operation cycles for Er0.52Sb2Te3. 

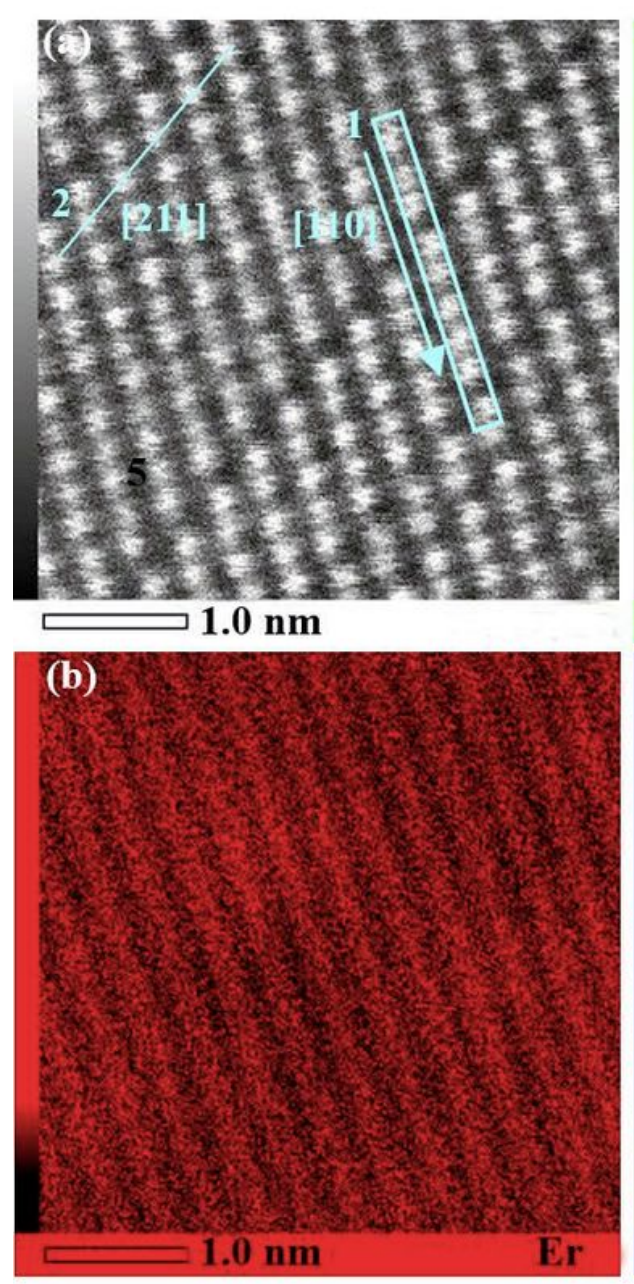
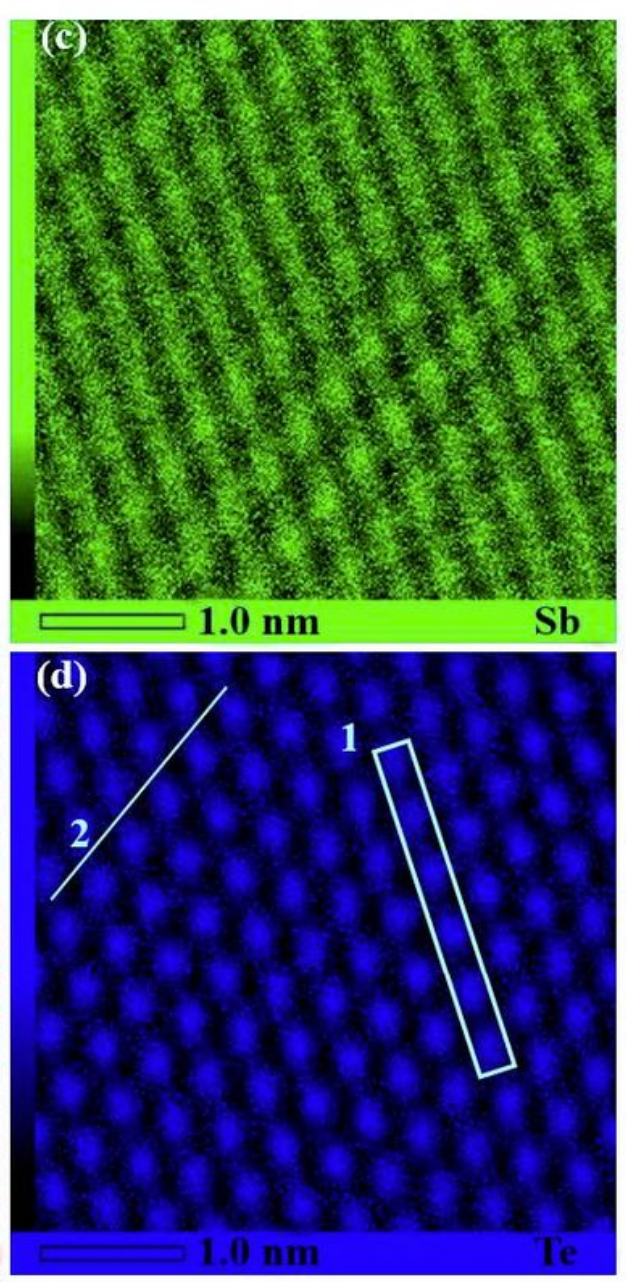
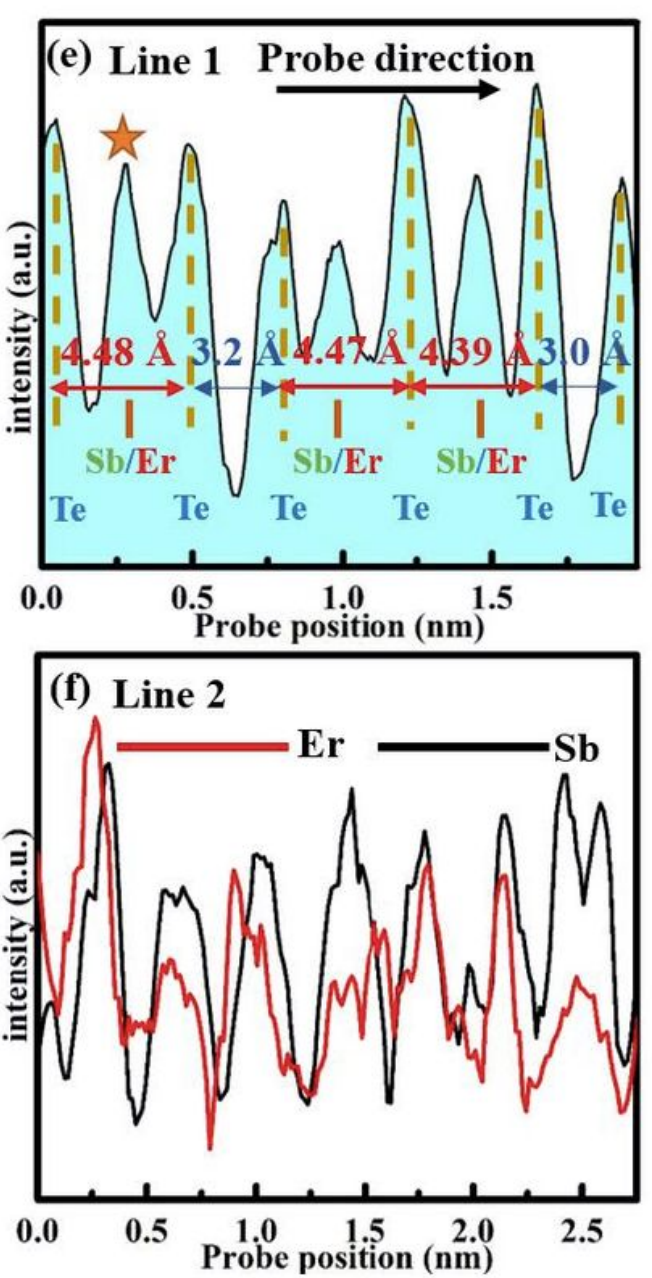

Figure 3

Atomic structure of cubic EST phase at $280^{\circ} \mathrm{C}$. (a), BF image. (b)-(d), the atomic resolution map images of $\mathrm{Er}$ (red), $\mathrm{Sb}$ (green) and Te (blue) atoms taken from (a). (e), the intensity profile of marked rectangle region in line 1. (f), the contrast intensity of Er and $\mathrm{Sb}$ along line 2. 

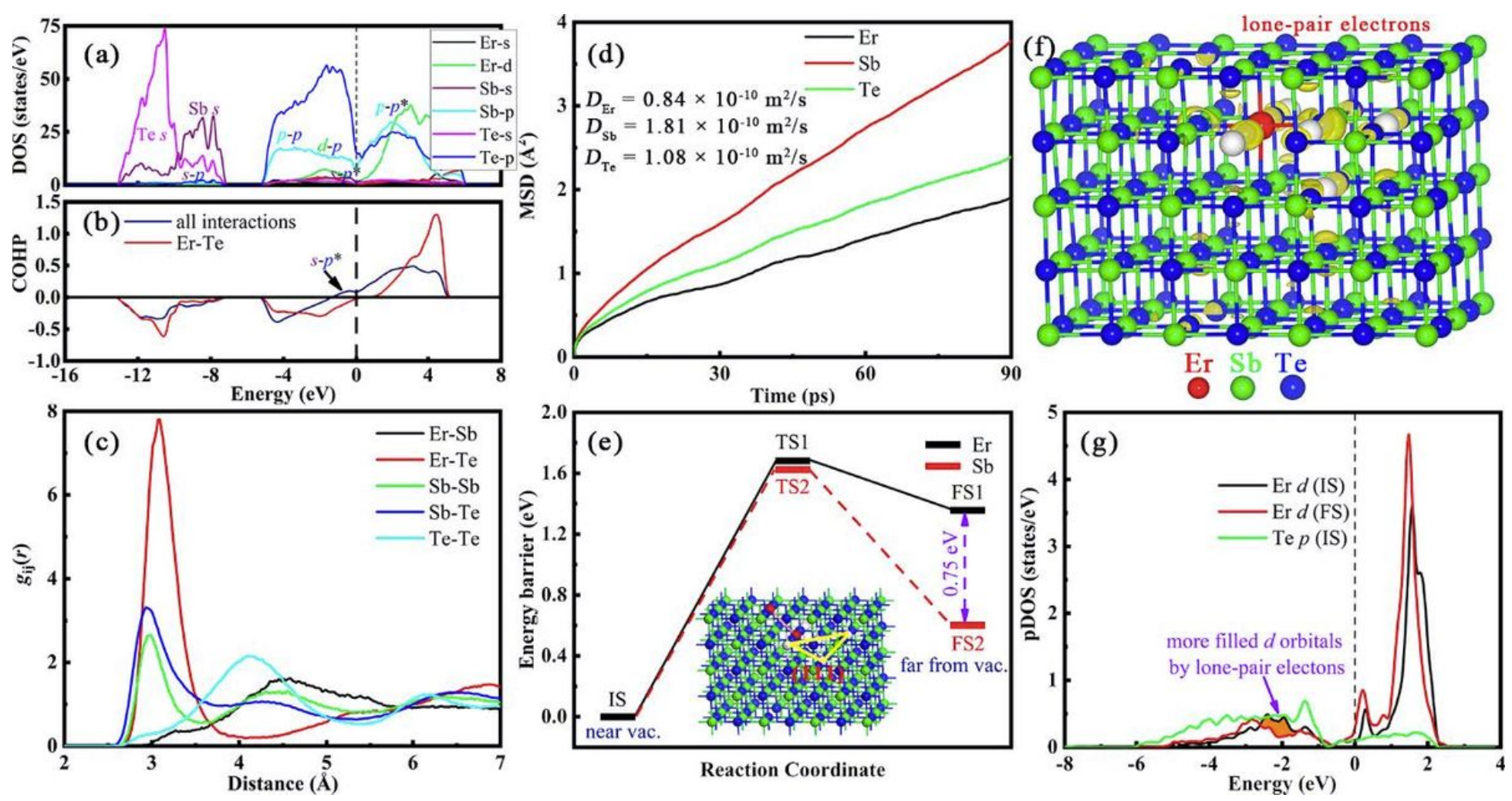

Figure 4

Evidences of stabilized Te atoms in both amorphous (a)-(d) and crystalline phases (e)-(g) by filling the empty d orbitals of Er atoms using the lone-pair electrons. (a), the pDOS of Er, Sb, and Te atoms with various orbitals. (b), the COHP of average values of all interactions and Er-Te interaction, respectively. (c), the PCFs of Er-Sb (black), Er-Te (red), Sb-Sb (green), Sb-Te (blue), and Te-Te (cyan) pairs. (d), the MSDs for Er, Sb, and Te atoms. (e), the energetic profile of diffusion barrier for Er (black) or Sb (red and dash), where a model with a four-vacancy-aggregated cluster in the (111) plane is shown in the inner graph. (f), the partial charge in the DOS region of $[-3,0] \mathrm{eV}$ in the IS structure, where 3D isosurface value is set as $0.2 \mathrm{eA}-3$. (g), the partial DOS of Er d (black) and Te p (green) orbitals in the IS structure, while the Er d (red) orbital in the FS structure also shown for comparison. It is noted that the pDOS of Te p orbitals is divided by Te number (48 herein). 

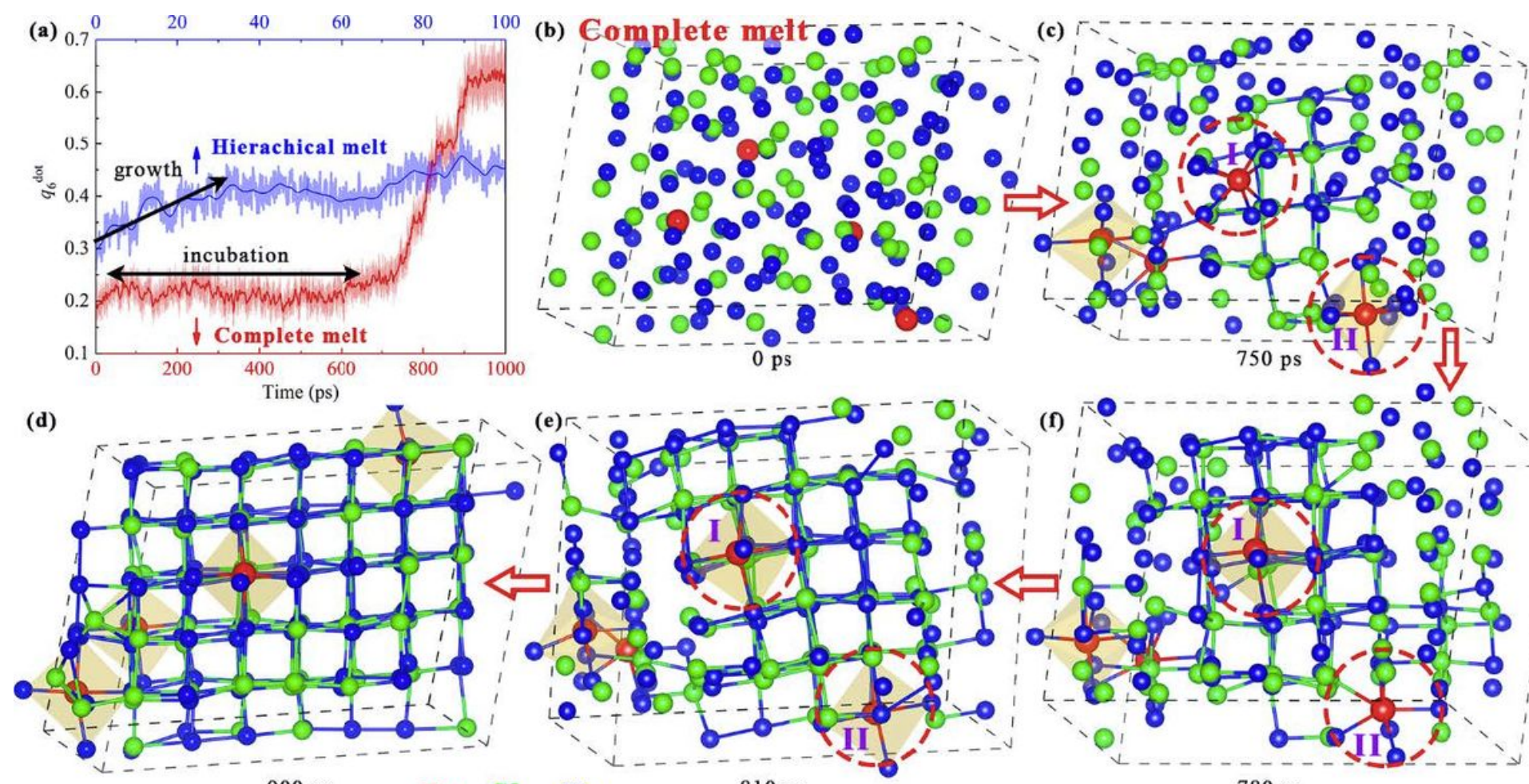

900 ps

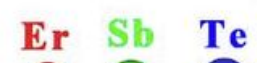

(e)
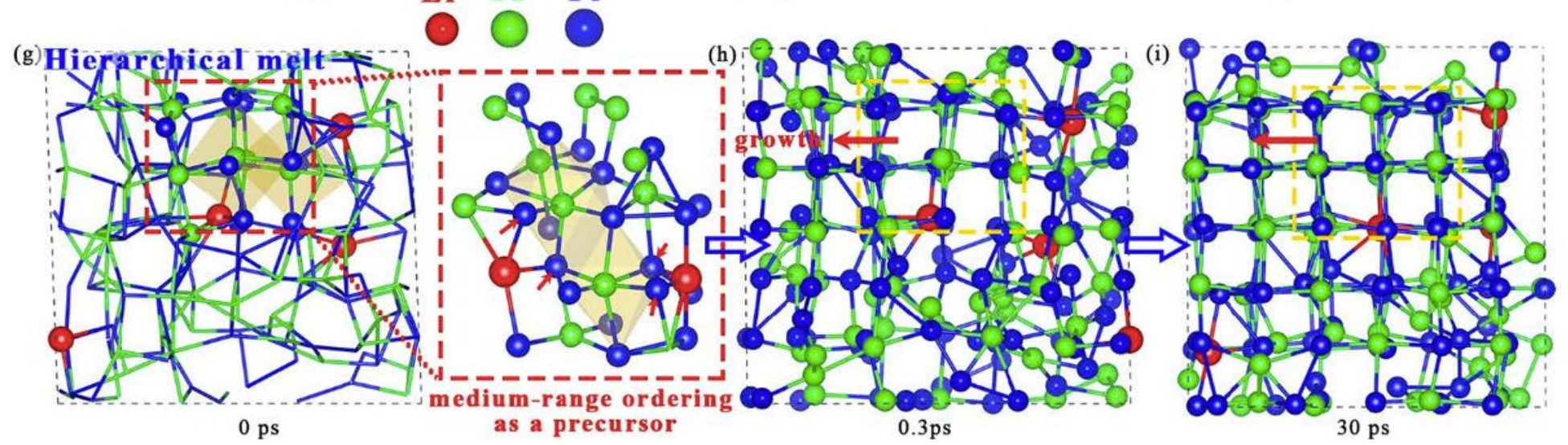

Figure 5

Hierarchical melt tuned crystallization. (a), the evolution of q6dot value during the crystallization for the complete melt (red) and hierarchical melt (blue) models. In the complete model, (b)-(f) show the snapshots at 0 ps, 750 ps, 780 ps, 810 ps, and 900 ps, respectively, where the octahedral environment around $\mathrm{Er}$ atoms is characterized by orange octahedral cages. The crystal-like atoms and their neighbor atoms are connected by bonds with the cutoff of $3.6 \AA$. The red circles in (c)-(e) emphasize the alterable Er local environments. In the hierarchical melt model, (g)-(i) show the snapshots at 0ps, 0.3ps, and 30ps, respectively.

\section{Supplementary Files}

This is a list of supplementary files associated with this preprint. Click to download.

- Supplementary.docx 